\title{
Análisis de las modificaciones en la estadificación del T según el manual del American Joint Committee on Cancer. ¿Cómo cambia nuestro manejo de los pacientes?
}

\author{
Estudio de corte transversal
}

\section{Analysis of the change in the staging of the T according to the manual of the American Joint Committee on Cancer. How does our patient management change?}

\section{Cross-sectional study}

Aníbal M. Parigini ${ }^{1}$, María Clara de Diego², Raúl Valdez ${ }^{2}$ y Corina Busso ${ }^{3}$ \begin{abstract}
RESUMEN
Antecedentes: En la octava edición del manual de estadificación del cáncer del American Joint Committee on Cancer (AJCC), se introdujeron cambios importantes en las categorías T, N y M. Al entrar en vigencia la octava guía de la AJCC, se modificó no solo el T, sino también la indicación de biopsia del ganglio centinela (BGC).

Entre los cambios más significativos en la estadificación se encuentran: la exclusión del índice mitótico (IM) de la categoría T en los melanomas finos (de hasta $1 \mathrm{~mm}$ de espesor) y el cambio del punto de corte para el espesor tumoral para discriminar un T1a ( $<0,8 \mathrm{~mm}$ sin ulceración) de unT1b ( $\geq 0,8 \mathrm{~mm}$ ). Objetivo: Comparar la estadificación inicial de los melanomas finos según el criterio utilizado en la séptima edición, con la que tendrían de acuerdo con la perspectiva actual del AJCC, con especial atención en el índice mitótico. Diseño y métodos: Estudio observacional, de corte transversal, realizado mediante la recolección de datos de las historias clínicas desde el 1 de enero de 2000 hasta el 31 de diciembre de 2017.

Resultados: De 131 melanomas finos incluidos, 28 tendrían cambios en su estadificación. Al considerar el nuevo punto de corte para el espesor tumoral, 22 melanomas T1a pasarían a T1b. Asimismo, se detectaron 20 melanomas con un $I M \geq 1$ mitosis $/ \mathrm{mm}^{2}$, de los cuales solo 6 tuvieron indicación de BGC por este criterio exclusivamente y serían clasificados como T1a en la actualidad. De estos, en 2 no se realizó la BGC por autodeterminación de los pacientes y en los 4 restantes el resultado fue negativo.

Conclusiones: Veintiocho de nuestros pacientes tendrían hoy diferencias en la indicación de BGC: 22 serían considerados con mayor riesgo de metástasis ganglionares y serían candidatos a su pesquisa. Los otros 6 pacientes ya no tendrían indicación de ese estudio por la baja posibilidad de encontrar metástasis ocultas, lo cual coincide con el resultado negativo de la BGC en los 4 pacientes que se sometieron al procedimiento.

Palabras clave: melanoma, estadificación, índice mitótico, Breslow, AJCC. Dermatol. Argent. 2020, 26 (1): 23-25
\end{abstract}

\section{ABSTRACT}

Background: In the 8th edition of the cancer staging manual of the American Joint Committee on Cancer (AJCC), important changes were made in the $T, N$ and $M$ categories. When the 8th guideline of the AJCC came into effect, not only was the Tstage modified, but also the indication for sentinel lymph node biopsy (SLNB).

The most significant changes in staging included: the exclusion of the mitotic index (MI) as a determinant of the T category and the change of the threshold of tumor thickness to discriminate a T1a $1<0.8 \mathrm{~mm}$ without ulceration) from a $T 1 b(\geq 0.8 \mathrm{~mm})$.

Objective: To compare the initial staging of thin melanomas according to the criteria used in the 7th edition, with the one that would have been used according to the current AJCC recommendations, with special focus on MI. Design and methods: Observational, cross-sectional study, carried out through the collection of data from medical records from January 1 , 2000 to December 31, 2017.
Results: There were 131 thin melanomas included, 28 of which would have had changes in their staging. When considering the modified threshold for tumor thickness, 22 T1a melanomas would be classified as T16. Among 20 thin melanomas with a MI $\geq 1$, only 6 had an indication for SLNB solely due to the MI criterion and would be now classified as T1a. Two of these did not undergo SLNB because they rejected the procedure, and in the remaining 4, there were no SLN metastasis.

Conclusions: Nowadays, 28 of our patients would have differences in the indication for SLNB: 22 would be considered to be at greater risk of lymph node metastasis and would be candidates for screening. The other 6 patients would no longer have an indication for this procedure due to the low probability of clinically occult metastases, which seems to concur with the negative result of SLNB in the 4 patients who underwent the procedure. Key words: melanoma, staging, mitotic index, Breslow, AJCC. Dermatol. Argent. 2020, 26 (1): 23-25 


\author{
1 Jefe de Residentes \\ 2 Médico de Planta \\ 3 Jefa del Servicio \\ Servicio de Dermatología, Hospital Universitario Austral, Pilar, \\ Buenos Aires, Argentina
}

Contacto del autor: Aníbal M. Parigini

Email: aparigin@cas.austral.edu.ar

Fecha de trabajo recibido: 15/10/2019

Fecha de trabajo aceptado: 31/1/2020

Conflicto de interés: los autores declaran que no existe conflicto de interés.

\section{INTRODUCCIÓN}

El melanoma cutáneo es una neoplasia cuya incidencia mundial está en aumento ${ }^{1}$. Su tratamiento y seguimiento, basados en una estadificación precisa, son necesarios para mejorar el pronóstico de los pacientes.

En la Unidad de Melanoma (UM) del Servicio de Dermatología del Hospital Universitario Austral, basamos nuestra guía de manejo y seguimiento de los pacientes con melanoma en las recomendaciones del manual de estadificación del cáncer del American Joint Committee on Cancer (AJCC).

En la reciente octava edición se introdujeron cambios importantes en las categorías T, N y M. Las modificaciones se fundamentaron en el análisis de una base de datos que incluía registros de pacientes con melanoma de 10 instituciones en los Estados Unidos, Europa y Australia ${ }^{2,3}$.

Uno de los cambios más significativos en la estadificación fue la exclusión del índice mitótico (IM) de la categoría $\mathrm{T}$ en los melanomas finos (de hasta $1 \mathrm{~mm}$ de espesor). Anteriormente, en la séptima edición, un $\mathrm{IM} \geq 1$ era uno de los criterios para diferenciar un tumor T1a $(\mathrm{IM}<1)$ de uno T1b (IM $\geq 1)$. Asimismo, se modificó la medición del espesor tumoral en milímetros (a un decimal) y el punto de corte para el espesor tumoral para discriminar un T1a ( $<0,8 \mathrm{~mm}$ sin ulceración) de un T1b $(\geq 0,8 \mathrm{~mm})$. Con respecto a la ulceración, esta se mantuvo como criterio de estadificación de la categoría T. Debido a estos cambios, se modificaron las indicaciones para la realización de la biopsia del ganglio centinela (BGC) (elemento usado principalmente para la estadificación) en los melanomas finos; se incluyeron melanomas de $0,8 \mathrm{~mm}$ o mayores, con independencia del IM o la ulceración, y se excluyeron melanomas menores de 0,8 con un IM de 1 o mayor ${ }^{2}$.

$\mathrm{Al}$ entrar en vigencia la nueva guía de la AJCC en enero de 2018, se modificó no solo el T, sino también la indicación de BGC. Por lo tanto, el objetivo de nuestro estudio fue comparar la estadificación inicial del melanoma fino determinada en su momento por la séptima guía de la AJCC, con la que se hubiera utilizado según las recomendaciones actuales del AJCC, con hincapié en el IM.

\section{OBJETIVO DE LA INVESTIGACIÓN}

Comparar las indicaciones de BGC en los melanomas finos tratados en la UM según el criterio utilizado antes de la implementación de la octava guía de la AJCC con el que se hubiera utilizado según las recomendaciones actuales de esa organización.

\section{Universo de estudio}

Se incluyeron pacientes con diagnóstico de melanoma fino desde el 1 de enero de 2000 hasta el 31 de diciembre de 2017, registrados en la historia clínica electrónica.

\section{Participantes}

Criterios de inclusión: pacientes con diagnóstico de melanoma cutáneo con espesor de Breslow $\leq 1 \mathrm{~mm}$ (melanoma fino).

Criterios de exclusión: melanoma in situ, metástasis ganglionares clínicamente evidentes o metástasis a distancia.

\section{MATERIALES Y MÉTODOS \\ Diseño}

Estudio observacional, de corte transversal.

\section{Variables del melanoma primario}

Todos los preparados histológicos fueron evaluados por dermatopatólogos con experiencia en lesiones melanocíticas. Se tuvieron en cuenta tanto las biopsias incisionales como las escisionales y, en caso de haber más de una biopsia, se incluyó para el análisis la que tuviera parámetros de mayor riesgo (mayor espesor tumoral, mayor IM y/o presencia de ulceración). Se evaluaron las siguientes características del tumor primario: espesor tumoral en milímetros, presencia de ulceración e IM en $\mathrm{mm}^{2}$, definido como la cantidad de mitosis presentes en un milímetro cuadrado a partir del denominado hot spot, es decir, el área de la dermis donde se encuentre el mayor número de mitosis, desde donde se extiende a los campos adyacentes hasta abarcar un milímetro cuadrado.

- Espesor: variable continua en milímetros.

- Ulceración: variable nominal dicotómica: sí/no.

- IM: variable nominal dicotómica: $<1 / \geq 1$ mitosis/ $\mathrm{mm}^{2}$.

- Biopsia del ganglio centinela variante nominal dicotómica: positivo/negativo (Foto 1).

A todos los pacientes evaluados se les realizaron estudios con técnicas de tinción de hematoxilina-eosina y, en caso de no detectar metástasis, se hicieron marcaciones de inmunohistoquímica con S-100, HMB45 y Melan A. 


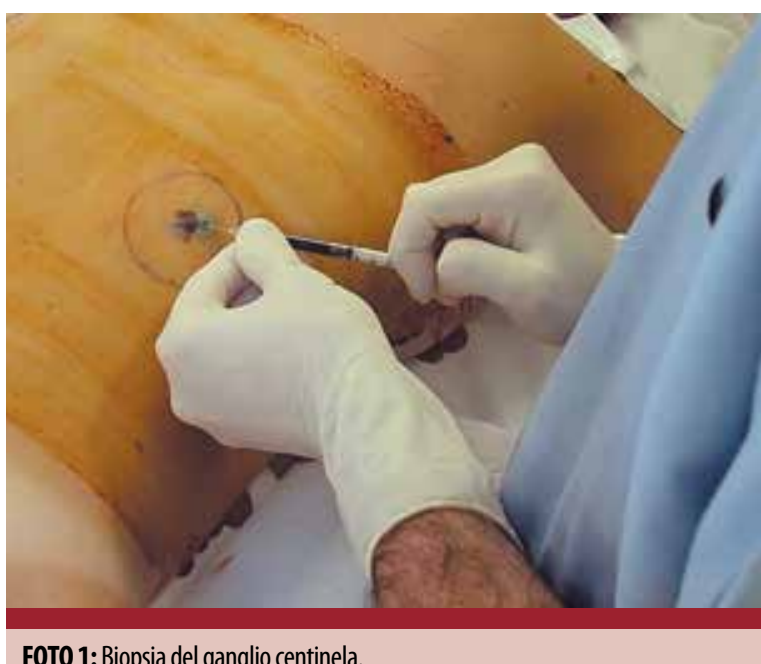

FOTO 1: Biopsia del ganglio centinela.

\section{RESULTADOS}

Se incluyeron 131 melanomas finos correspondientes a un número igual de pacientes. En 103 casos no hubo diferencias en la estadificación del T, mientras que en 28 pacientes existió un cambio: 22 presentaban un T1a en la séptima edición de la AJCC y un T1b en la octava, de acuerdo con el cambio en el valor de corte del espesor de Breslow; los 6 pacientes restantes estaban clasificados en la séptima edición como T1b por presentar un IM mayor o igual a 1 , mientras que en la octava serían catalogados como T1a (Gráfico 1).

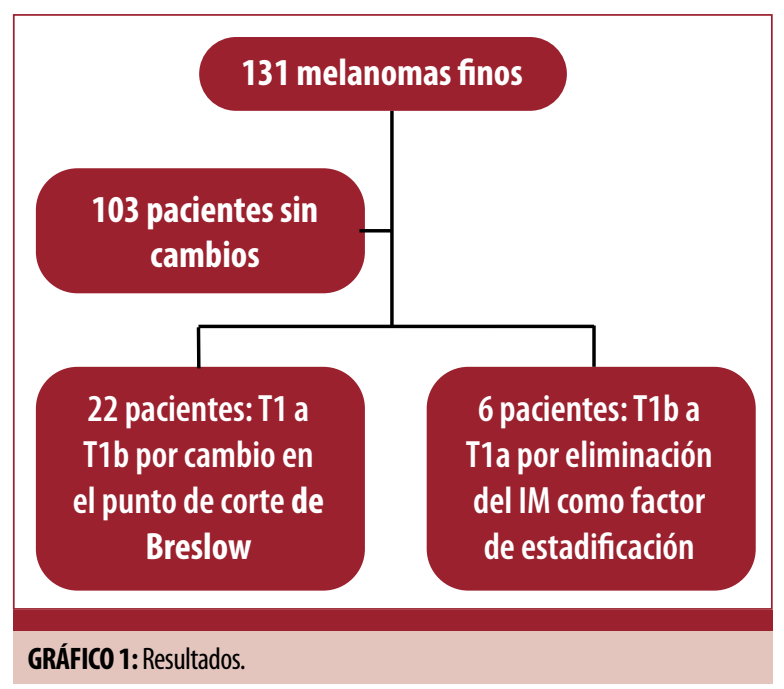

\section{BIBLIOGRAFÍA}

1. The American Cancer Society medical and editorial content team. Key Statistics for Melanoma Skin Cancer. Disponible en http:// www.cancer.org/cancer/melanoma-skin-cancer/about/keystatistics.html\#references. [Consulta octubre 2019]

2. Gershenwald JE, Scolyer RA, Hess KR, Sondak V, et ál. Melanoma staging: Evidence-based changes in the American Joint Committee on Cancer eighth edition cancer staging manual. CA Cancer J Clin 2017;67:472-492.
Con respecto específicamente al IM, 20 pacientes presentaron un $I M \geq 1$. De estos, solo 6 tuvieron como único criterio de BGC el IM según la séptima edición, mientras que por la octava no tenían indicación y hubieran sido clasificados como T1a. De los 6 mencionados, en 2 casos no se realizó la biopsia por voluntad del paciente y, en los 4 restantes, esta fue negativa para metástasis.

\section{CONCLUSIONES}

La correcta estadificación del melanoma es fundamental para definir la conducta terapéutica y determinar el pronóstico. La eliminación del IM y la modificación del punto de corte del espesor en los melanomas finos conllevan diferencias en la identificación de qué pacientes tienen mayor riesgo de enfermedad metastásica ganglionar.

Veintiocho de nuestros pacientes diagnosticados con melanoma fino a lo largo de 8 años tendrían hoy diferencias en la indicación de BGC: 22 serían considerados con mayor riesgo de metástasis ganglionares y serían candidatos a su pesquisa. Los otros 6 pacientes ya no tendrían indicación de ese estudio por la baja posibilidad de encontrar metástasis ocultas, lo cual coincide con el resultado negativo de la BGC en los 4 pacientes que se sometieron al procedimiento.

En la actualidad, la BGC se utiliza para hacer una estadificación precisa del melanoma. En los últimos años se ha cuestionado la realización de la linfadenectomía total profiláctica ante el hallazgo de metástasis en el ganglio centinela; sin embargo, conocer el grado de compromiso de este último guía la toma de decisiones terapéuticas, incluida la indicación de tratamiento adyuvante. Además, determina la frecuencia y el tipo de seguimiento que requiere cada paciente ${ }^{4}$.

\section{Agradecimiento}

Al Dr. Pedro Valdez, cirujano de cabeza y cuello, por la imagen de la biopsia del ganglio centinela.

3. Balch CM, Gershenwald JE, Soong SJ, Thompson JF, et ál. Final version of 2009 AJCC melanoma staging and classification. J Clin Oncol 2009;27:6199-6206.

4. Faries MB, Thompson JF, Cochran AJ, et ál. Completion dissection or observation for sentinel-node metastasis in melanoma. N Engl J Med 2017;376:2211-2222. 